\title{
A Multisegmented Polystyrene with pH-Cleavable Linkages
}

\author{
Tae-Hyeon Kang and Hyung-il Lee* \\ Department of Chemistry, University of Ulsan, Ulsan 680-749, Korea. *E-mail: sims0904@ulsan.ac.kr \\ Received March 3, 2014, Accepted May 12, 2014
}

\begin{abstract}
A multisegmented polystyrene (PS) with pH-cleavable ester and carbamate linkages was successfully synthesized by a combination of atom transfer radical polymerization (ATRP) and $\mathrm{Cu}$ (I)-catalyzed 1,3-dipolar cycloaddition of azide and alkynes (click chemistry). ATRP was employed to synthesize polystyrene from hydroxyl-terminated initiator using $\mathrm{CuBr} / N, N, N^{\prime}, N^{\prime \prime}, N^{\prime \prime}$-pentamethyldiethylenetriamine (PMDETA) as the catalyst. The reaction of the resulting PS with sodium azide yielded the azido-terminated polymer. The hydroxyl group in the other end of the polymer was reacted with 4-nitrophenyl chloroformate (NPC), followed by reaction with propargylamine to produce an alkyne end group with a carbamate linkage. The PS with an alkyne group in one end and an azide group in the other end was then self-coupled in the presence of $\mathrm{CuBr} / 2,2^{\prime}-$ bipyridyl (bpy) in DMF to yield a desired multisegmented PS. Molecular weight and molecular weight distribution of the self-coupled polymer increased with time, as in the typical step-growth-type polymerization processes. Finally, we demonstrated that the ester and carbamate linkages of the multisegmented PS were hydrolyzed in the presence of $\mathrm{HCl}$ to yield individual PS chains.
\end{abstract}

Key Words : ATRP, Multisegmented, Click reactions, Degradable, Polystyrene

\section{Introduction}

Multisegmented polymers consist of successive polymer blocks that are connected by a linker. ${ }^{1,2}$ The use of a labile linker can lead to degradable multisegmented polymers. They exhibit quite different properties when compared to their homopolymers. ${ }^{3}$ Degradable multisegmented polymers would find potential applications in the field of green chemistry and drug delivery. ${ }^{4}$ Due to these unusual architectural features, materials with new morphologies and improved properties can be obtained. In order to prepare multisegmented polymers, the robust techniques for polymer synthesis and the use of highly selective and efficient modification reactions are required.

Controlled radical polymerization (CRP) became one of the powerful methods for polymer synthesis owing to the ability to polymerize a wide range of commercially available monomers to yield well-defined polymers with designed architecture. ${ }^{5-12}$ The 1,3-dipolar azide-alkyne cycloaddition, one of click reactions, has been rapidly expanding as a method for chemical transformation and synthesis of novel materials. ${ }^{13,14}$ This reaction is combined particularly well with controlled radical polymerization (CRP) techniques such as atom transfer radical polymerization (ATRP) ${ }^{15-17}$ since it can be easily employed to prepare functional polymers from ATRP products with high degree of end functionality. ${ }^{18}$ The halogen end group present on a polymer prepared by ATRP is efficiently displaced by an azide group through nucleophilic substitution using sodium azide.

In this paper, a novel synthesis to prepare degradable multisegmented polystyrene (PS) with hydrolyzable ester and carbamate linkages via ATRP and click reaction is presented. Acid-catalyzed degradation process is reported as well.

\section{Experimental}

Material. Styrene (95\%, Sigma) was purified by passing through a column filled with basic alumina (Acros) to remove inhibitors. Triethylamine (TEA, 99.5\%), sodium azide (99.5\%), dichloromethane (DCM, 99.9\%), N,N-dimethylformamide (DMF, 99.8\%), ethylene glycol (99.8\%), 2-bromoisobutyryl bromide (98\%), propargylamine (98\%), 4-nitrophenyl chloroformate $(96 \%)$, and $\mathrm{CuBr}(98 \%)$ were purchased from Aldrich with the highest purity and were used as received without further purification. Anisole (99\%), 2,2'-bipyridyl (bpy, 99\%), and $N, N, N^{\prime}, N^{\prime \prime}, N^{\prime \prime}$-pentamethyldiethylenetriamine (PMDETA, 95\%) were purchased from Tokyo Chemical Industry (TCI) and used as received.

Instrumentation. ${ }^{1} \mathrm{H}-\mathrm{NMR}$ spectra were collected in $\mathrm{CDCl}_{3}$ on a Bruker avance $300 \mathrm{MHz}$ NMR spectrometer. The apparent molecular weights and molecular weight distributions were measured by gel permeation chromatography (GPC, Agilent technologies 1200 series) using a polystyrene (PS) standard, with DMF as the eluent at $30^{\circ} \mathrm{C}$ and a flow rate of $1.00 \mathrm{~mL} / \mathrm{min}$. Thermal characterization (glass transition temperature) of homo PS and multisegmented PS (4-7 mg) was carried out with the aid of TA Instrument Q20 operated at a heating rate $10^{\circ} \mathrm{C} / \mathrm{min}$. Thermogravimetric analysis (TGA) was carried out with the aid of TA Instrument Q50.

2-Hydroxyethyl 2-Bromoisobutyrate. Ethylene glycol $(25.0 \mathrm{~mL}, 448.5 \mathrm{mmol})$, triethylamine (TEA, 62.5 mL) and THF $(1000 \mathrm{~mL})$ were added to round-bottomed flask and immersed into an ice-water bath. 2-Bromoisobutyryl bromide (44.3 mL, $358.4 \mathrm{mmol}$ ) in $40 \mathrm{~mL}$ THF was added drop-wise into the mixture within $10 \mathrm{~min}$. The reaction mixture was allowed to stir for overnight at room temperature, allowing insoluble salt to precipitate out of the solution. After filtration, 
the solvent was evaporated. The product was purified by column chromatography using Hexane-EA (1:1) as an eluent to give the product as pale yellow liquid $(15 \mathrm{~mL}, 20 \%)$. ${ }^{1} \mathrm{H}$ NMR $\left(300 \mathrm{MHz}, \mathrm{CDCl}_{3}\right) \delta$ was as follows: $4.32-4.28(2 \mathrm{H}, \mathrm{t}$, $\left.\mathrm{HOCH}_{2} \mathrm{CH}_{2}\right) ; 3.91-3.81\left(2 \mathrm{H}, \mathrm{t}, \mathrm{HOCH}_{2} \mathrm{CH}_{2}\right) ; 3.00-2.60(1 \mathrm{H}, \mathrm{s}$, $\left.\mathrm{HOCH}_{2} \mathrm{CH}_{2}\right) ; 1.96-1.89\left(6 \mathrm{H}, \mathrm{s},(\mathrm{C}=\mathrm{O}) \mathrm{C}\left(\mathrm{CH}_{3}\right)_{2} \mathrm{Br}\right)$.

HO-Polystyrene-Br (HO-PS-Br). A clean and dry Schlenk flask was charged with styrene $(5.0 \mathrm{~mL}, 43.64 \mathrm{mmol})$, PMDETA (45.56 $\mu \mathrm{L}, 0.22 \mathrm{mmol})$, anisole $(5.0 \mathrm{~mL})$, and 2hydroxyethyl 2-bromoisobutyrate $(46.05 \mathrm{mg}, 0.22 \mathrm{mmol})$. The flask was deoxygenated by five freeze-pump-thaw cycles. $\mathrm{CuBr}(31.3 \mathrm{mg}, 0.22 \mathrm{mmol})$ was added to the frozen mixture in the presence of argon. The flask was filled with argon and heated in an oil bath at $110^{\circ} \mathrm{C} .5 \mathrm{~h}$ later, when monomer conversion reached $60 \%$, polymerization was stopped by exposing the solution to air. The resulting solution was passed through neutral alumina to remove the copper complex, and the polymer was precipitated twice in methanol and dried under vacuum at room temperature for 24 h. $\mathrm{M}_{\mathrm{n}}=12800 \mathrm{~g} / \mathrm{mol}, \mathrm{M}_{\mathrm{w}} / \mathrm{M}_{\mathrm{n}}=1.07$.

Azide-Modified Polystyrene (HO-PS-N 3 ). $\mathrm{NaN}_{3}(28.6$ $\mathrm{mg}, 0.44 \mathrm{mmol})$, HO-PS-Br (1.5 g, $0.22 \mathrm{mmol})$, and DMF $(15 \mathrm{~mL})$ were added to a $50 \mathrm{~mL}$ round bottom flask and stirred for $48 \mathrm{~h}$ at room temperature, allowing insoluble salt to precipitate out of solution. After filtration, the polymer was precipitated into methanol and dried under vacuum at room temperature for $24 \mathrm{~h}$.

NPC-Modified Polystyrene (NPC-PS-N ${ }_{3}$ ). HO-PS-N 3 was activated by 4-nitrophenyl chloroformate (p-NPC). 1.3 $\mathrm{g}(0.22 \mathrm{mmol})$ of HO-PS- $\mathrm{N}_{3}$ and triethylamine $(0.097 \mathrm{~mL}$, $0.66 \mathrm{mmol})$ were dissolved in $15 \mathrm{~mL}$ anhydrous dichloromethane at $0{ }^{\circ} \mathrm{C}$. p-NPC $(0.089 \mathrm{~g}, 0.44 \mathrm{mmol})$ dissolved in $10 \mathrm{~mL}$ anhydrous dichloromethane was added dropwise into the mixed solution. The reaction proceeded for $2 \mathrm{~h}$ at $0{ }^{\circ} \mathrm{C}$, and then at room temperature for $24 \mathrm{~h}$ with stirring. After concentration, the product, NPC-PS-N $\mathrm{N}_{3}$, was obtained by precipitation in methanol and dried in vacuum at room temperature for $24 \mathrm{~h}$.

Alkyne-Modified Polystyrene (Alkyne-PS-N ${ }_{3}$ ). NPC$\mathrm{PS}^{-N_{3}}(1.0 \mathrm{~g}, 0.22 \mathrm{mmol})$ dissolved in dichloromethane
$(15 \mathrm{~mL})$ was added into the propargylamine $(28.2 \mu \mathrm{L}$, $0.44 \mathrm{mmol})$ solution in dichloromethane $(3 \mathrm{~mL})$ and reacted for $24 \mathrm{~h}$ at room temperature. After the evaporation of dichloromethane, the product was dissolved in $10 \mathrm{~mL}$ dichloromethane. After concentration, the product, alkyne-PS-N $\mathrm{N}_{3}$, was obtained by precipitation in methanol and dried in vacuum at room temperature for $24 \mathrm{~h}$.

CuBr-Catalyzed Click Coupling of $\alpha$-Alkyne- $\omega$-azidoterminated Polystyrene (Multisegmented PS). AlkynePS-N $_{3}(0.1 \mathrm{~g}, 0.05 \mathrm{mmol})$, dry DMF $(4 \mathrm{~mL})$, and bpy $(7.8$ $\mathrm{mg}, 0.05 \mathrm{mmol})$ were added to a Schlenk flask, and the vessel was subjected to three vacuum-nitrogen cycles. $\mathrm{CuBr}$ (3.6 mg, $0.05 \mathrm{mmol}$ ) was added, and the mixture was allowed to stir at room temperature for $24 \mathrm{~h}$. The final product was obtained by precipitation in methanol and dried in vacuum at room temperature for $24 \mathrm{~h}$.

Degradation of Multisegmented PS by Hydrolysis Reaction. To the clicked multisegmented PS $(0.01 \mathrm{~g})$ in DMF (3 $\mathrm{mL}$ ) was added 5 drops of $2 \mathrm{M} \mathrm{HCl}$ solution. The reaction mixture was stirred at $100{ }^{\circ} \mathrm{C}$ for $6 \mathrm{~h}$, precipitated into methanol, and dried under vacuum at room temperature for $24 \mathrm{~h}$.

\section{Results and Discussion}

The strategy employed in this study is schematically illustrated in Scheme 1. ATRP was employed to synthesize styrene using a $\mathrm{CuBr} / \mathrm{PMDETA}$ catalyst system with a hydroxy-terminated initiator, 2-hydroxyethyl 2-bromoisobutyrate. The ATRP reaction was stopped at relatively low monomer conversion $(60 \%)$ to ensure a high degree of bromine end functionality. The molecular weight and molecular weight distribution of the resulting HO-PS-Br were obtained using a GPC DMF line with polystyrene (PS) standards $\left(M_{n}=12800 \mathrm{~g} / \mathrm{mol}, M_{w} / M_{n}=1.07\right)$. As shown in Figure 1, the GPC traces showed monomodal distributions with the relatively low molecular weight distribution, indicating good control over the polymerization. The apparent molecular weight $(12,800 \mathrm{~g} / \mathrm{mol})$ matched well with the theoretical molecular weight calculated from the styrene conversion $\left(\mathrm{M}_{\mathrm{n} \text {,theory }}=\right.$ conversion $\times \mathrm{MW}_{\text {styrene }} \times[\text { styrene }]_{0} /$

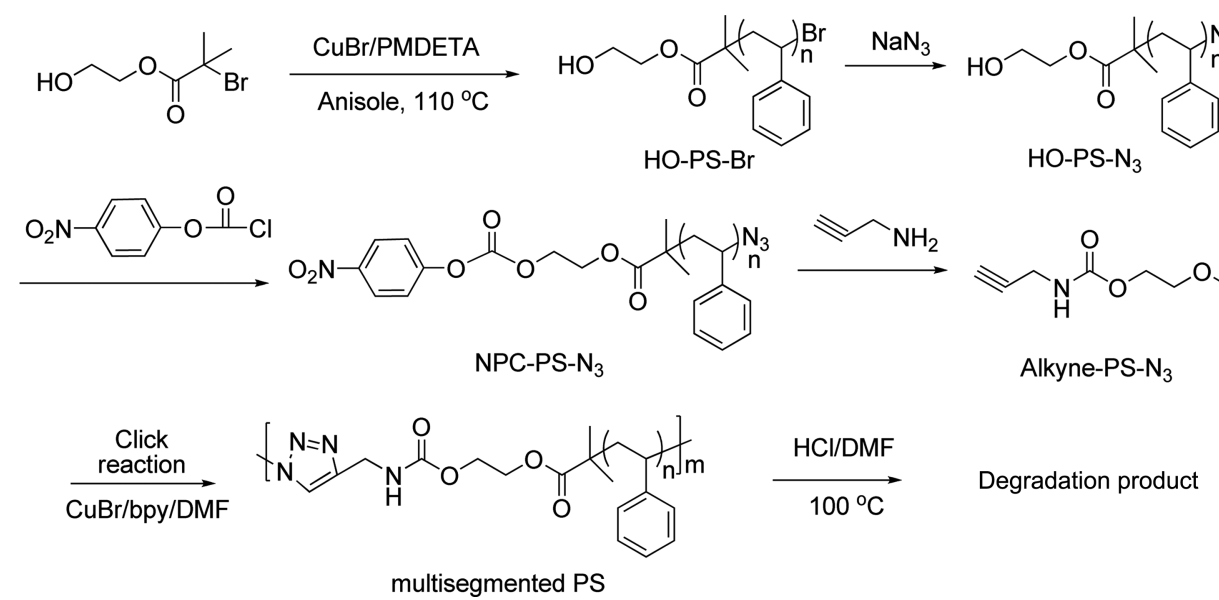

Scheme 1. Synthesis of multisegmented PS prepared by a combi- nation of ATRP of styrene and click reaction. 


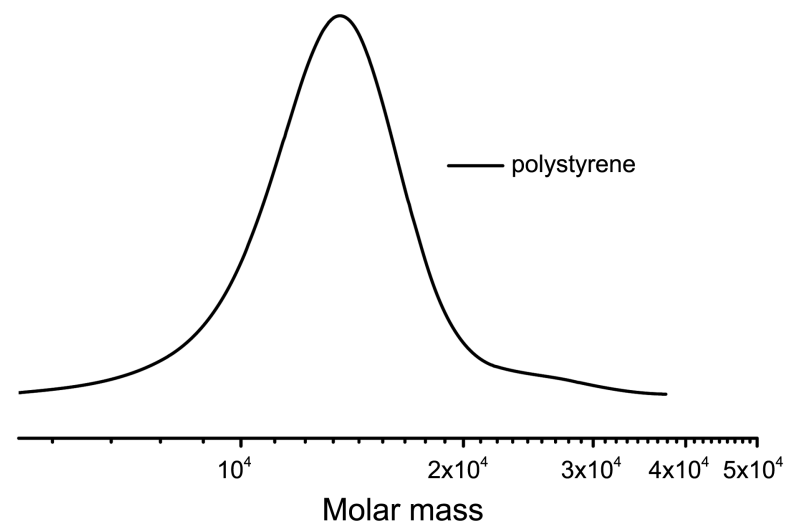

Figure 1. DMF GPC traces of HO-PS-Br.

[2-hydroxyethyl 2-bromoisobutyrate $]_{0}=0.6 \times 10^{4} \mathrm{~g} / \mathrm{mol} \times$ $200=12,500 \mathrm{~g} / \mathrm{mol}$ ).

The resulting HO-PS-Br was reacted with sodium azide to yield the corresponding azido-terminated polymer, HO-PS$\mathrm{N}_{3}$. The reaction was carried out in DMF at room temperature. Two-fold molar excess of sodium azide over chain end bromine groups was used to ensure the quantitative transformation. The ${ }^{1} \mathrm{H}-\mathrm{NMR}$ spectra provided the evidence of the successful introduction of azido group by nucleophilic substitution (Figure 2). It was monitored that a signal from 4.30 to 4.60 ppm due to the methine proton neighboring the bromine chain end (e) disappeared while a new peak (f: from 4.80 to $4.10 \mathrm{ppm}$ ) originaed from the methine proton neighboring the azido chain end appeared. Next, the hydorxy groups in the other end of polymer were reacted with p-NPC to yield
NPC-PS-N $\mathrm{N}_{3}$. The reaction was carried out in dichloromethane in the presence of trimethylamine at room temperature. Twofold molar excess of p-NPC over chain end hydorxy groups was employed to achieve the quantitative reaction. The successful transformation was monitored by ${ }^{1} \mathrm{H}-\mathrm{NMR}$ spectra (Figure 2). It was shown that signals at 7.35 and $8.25 \mathrm{ppm}$ due to the aromatic protons of NPC chain end ( $\mathrm{g}$ and $\mathrm{h}$ ) appeared. Alkyne groups were then introduced by replacing NPC groups by propargylamine. This reaction was quantitative as verified by ${ }^{1} \mathrm{H}-\mathrm{NMR}$ spectra (Figure 2). Signals at 7.35 and $8.25 \mathrm{ppm}$ due to the aromatic protons of NPC chain end disappeared completely. Finally, the resulting alkyne-PS-N 3 was subsequently subjected to click reaction, leading to selfcoupling. Even though $\mathrm{CuBr}$ exhibits sufficient solubility in DMF, extra ligand was added to achieve efficient coupling. The reaction was carried out under an argon atmosphere to avoid air oxidation of the $\mathrm{CuBr}$. The successful click coupling reaction was proved by ${ }^{1} \mathrm{H}-\mathrm{NMR}$ spectra (Figure 2 ). The signal due to the methine proton neighboring the azido group (f : from 4.80 to $4.10 \mathrm{ppm}$ ) completely disappeared while a new peak (i : from 4.95 to $5.10 \mathrm{ppm}$ ) appeared, which was attributed to the methine proton neighboring the triazole ring. No methine proton neighboring the azido group was observed in the ${ }^{1} \mathrm{H}-\mathrm{NMR}$ spectrum of the final polymer, proving the high efficiency of click coupling reaction. The step-growth click coupling reaction was further characterized by GPC. Figure 3 shows the evolution of the GPC traces with time. The click coupling of the parent alkyne-PS-N3 resulted in a multisegmented PS with high molecular weight $\left(\mathrm{M}_{\mathrm{n}}=\right.$ $23000 \mathrm{~g} / \mathrm{mol})$ and broad molecular weight distribution $\left(\mathrm{M}_{\mathrm{w}} /\right.$
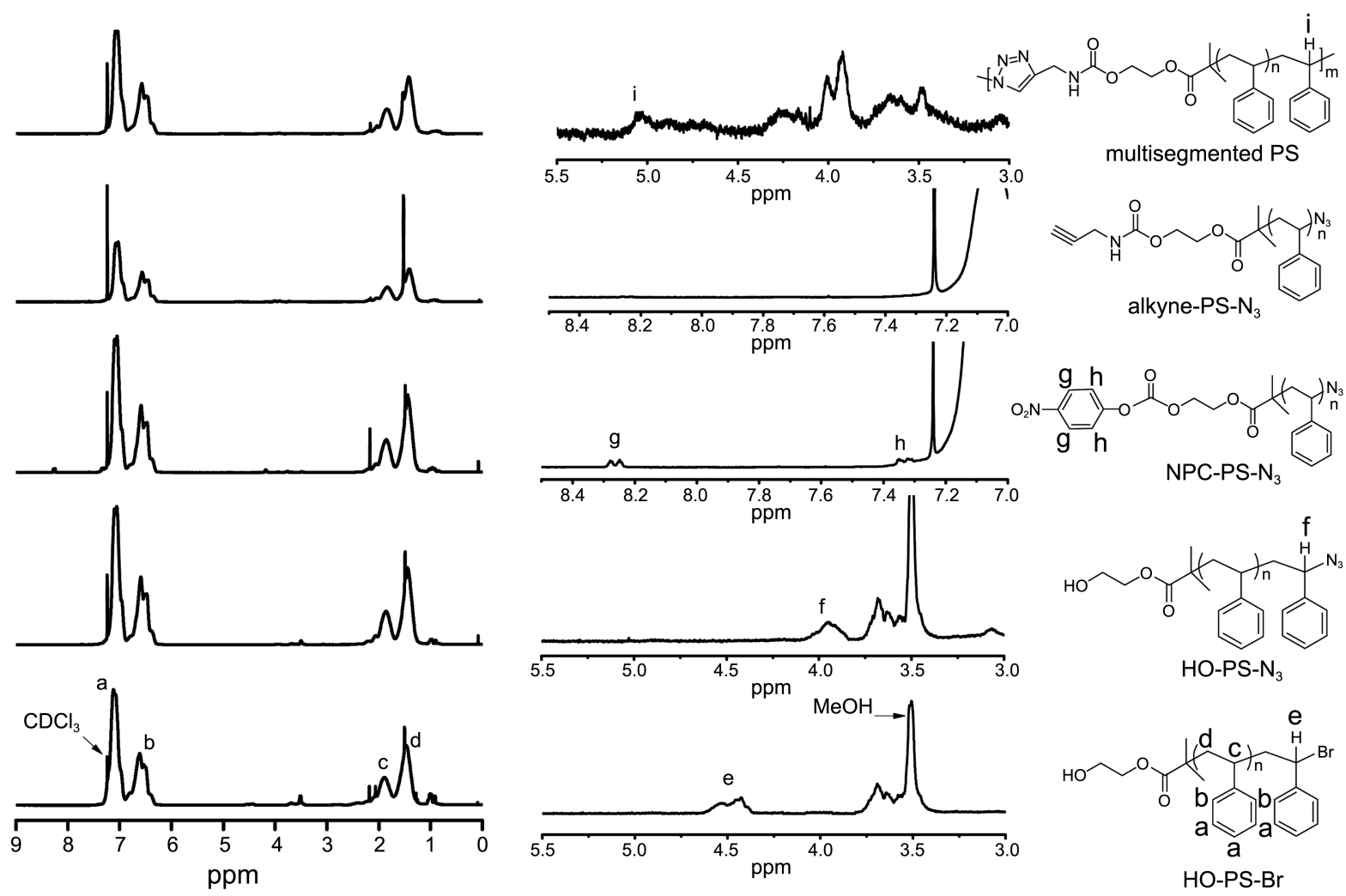

Figure 2. ${ }^{1} \mathrm{H}-\mathrm{NMR}$ spectra of HO-PS-Br, HO-PS-N3, NPC-PS-N3, alkyne-PS-N3, and multisegmented PS. 


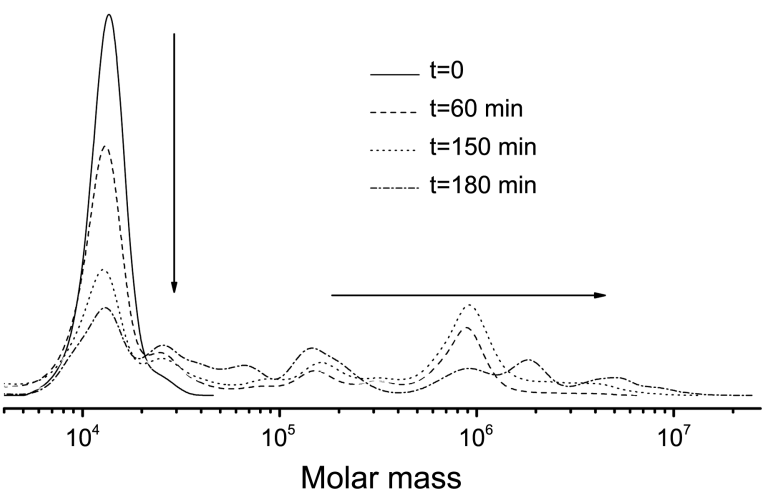

Figure 3. Evolution of the GPC traces during step-growth click coupling of alkyne-PS-N $\mathrm{N}_{3}$, leading to multisegmented PS. The arrows indicate that starting homopolymer was consumed while the generation of multisegmented PS.

$M_{n}=3.96$ ), as expected for a step-growth process. It seemed that not all of the starting homopolymer was consumed but the considerable amount of high molecular weight coupling product was generated. About $15 \%$ of the starting polymer, alkyne-PS-N3, remained after $180 \mathrm{~min}$, which was deduced by calculating the relative area of low molecular weight peak from the total area of the GPC trace. No further change in the GPC trace was observed even after prolonged period of the reaction time.

Thermogravimetric analysis (TGA) and differential scann-
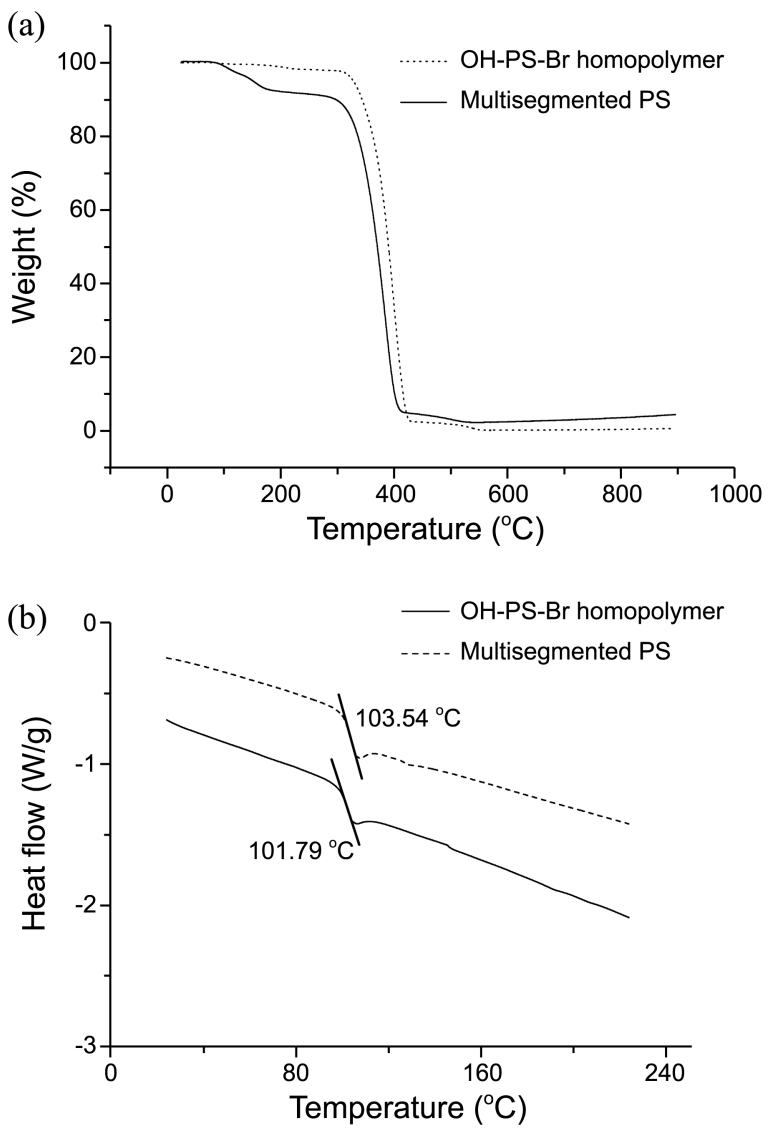

Figure 4. (a) TGA and (b) DSC thermograms HO-PS-Br and multisegmented PS.

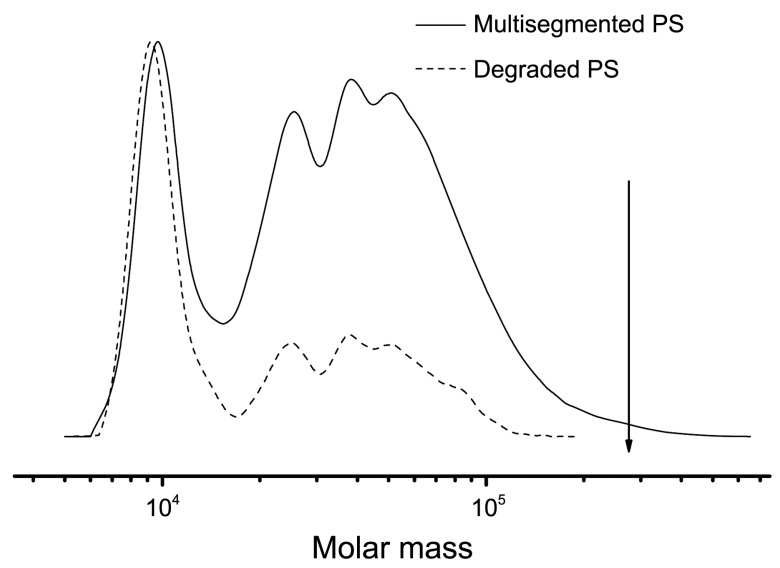

Figure 5. GPC traces of multisegmented PS (before hydrolysis) and degraded PS (after hydrolysis). The arrows indicate the progress of degradation.

ing calorimetry (DSC) were carried out to compare thermal properties of HO-PS-Br and multisegmented PS. As shown in Figure 4(a), the multisegmented PS showed a mass loss of $10 \%$ upon heating to $320^{\circ} \mathrm{C}$, which might be attributed to thermally labile ester and carbamate groups contained in multisegmented PS. We have also investigated DSC of the HO-PS-Br and multisegmented PS (Figure 4(b)), however, we could observe a subtle $1.75^{\circ} \mathrm{C}$ difference of the glass transition temperature $\left(T_{\mathrm{g}}\right)$ between two samples Hence, the multisegmented structure has a minimal effect on the $T_{\mathrm{g}}$.

The resulting multisegmented PS was subjected to degradation, leading to smaller molecular weight products. Acidcatalyzed hydrolysis was performed in DMF with excess amount of $\mathrm{HCl}$ for $24 \mathrm{~h}$. Figure 5 the evolution of the GPC traces before and after the hydrolysis reaction. After acidcatalyzed hydrolysis, multisegmented PS was degraded into smaller species with lower molecular weight $\left(\mathrm{M}_{\mathrm{n}}=11000 \mathrm{~g} /\right.$ mol) and broad molecular weight distribution $\left(\mathrm{M}_{\mathrm{w}} / \mathrm{M}_{\mathrm{n}}=\right.$ 3.55). Not all of the starting multisegmented PS was degraded. It was, however, clearly seen that the amount of high molecular weight polymers decreased substantially after hydrolysis. Since there are two hydrolyzable groups, ester and carbamate, this hydrolysis reaction was facilitated.

\section{Conclusions}

We demonstrated the synthesis of a multisegmented PS with $\mathrm{pH}$-cleavable ester and carbamate linkages in the main chain via a combination of atom transfer radical polymerization (ATRP) and $\mathrm{Cu}(\mathrm{I})$-catalyzed 1,3-dipolar cycloaddition of azide and alkynes. Homo PS polymer with ester and carbamate linkages was self-coupled by step-growth click reaction to yield a multisegmented PS containing 1,2,3-triazole linkages. The successful synthesis of a multisegmented PS was verified by ${ }^{1} \mathrm{H}-\mathrm{NMR}$ spectroscopy and GPC. The resulting multisegmented PS was degraded to smaller molecular weight products in the presence of $\mathrm{HCl}$. In conclusion, we designed a unique system to prepare multisegmented polymers that can be easily degraded by simple acid-catalyzed hydrolysis. 
Acknowledgments. This work was supported by University of Ulsan Research Fund of 2012.

\section{References}

1. Tsarevsky, N. V.; Sumerlin, B. S.; Matyjaszewski, K. Macromolecules 2005, 38, 3558-3561.

2. Nicolay, R.; Marx, L.; Hemery, P.; Matyjaszewski, K. Macromolecules 2007, 40, 9217-9223.

3. Liu, R. C. W.; Pallier, A.; Brestaz, M.; Pantoustier, N.; Tribet, C. Macromolecules 2007, 40, 4276-4286.

4. Li, X.; Liu, K. L.; Li, J.; Tan, E. P. S.; Chan, L. M.; Lim, C. T.; Goh, S. H. Biomacromolecules 2006, 7, 3112-3119.

5. Braunecker, W. A.; Matyjaszewski, K. Prog. Polym. Sci. 2007, 32, 93-146.

6. Goto, A.; Fukuda, T. Prog. Polym. Sci. 2004, 29, 329-385.

7. Lowe, A. B.; McCormick, C. L. Prog. Polym. Sci. 2007, 32, 283351.
8. Matyjaszewski, K. Prog. Polym. Sci. 2005, 30, 858-875.

9. Coessens, V.; Pintauer, T.; Matyjaszewski, K. Prog. Polym. Sci. 2001, 26, 337-377.

10. Hawker, C. J.; Bosman, A. W.; Harth, E. Chem. Rev. 2001, 101, 3661-3688.

11. Kamigaito, M.; Ando, T.; Sawamoto, M. Chem. Rev. 2001, 101, 3689-3746.

12. Tsarevsky Nicolay, V.; Matyjaszewski, K. Chem. Rev. 2007, 107, 2270-2299.

13. Lutz, J.-F. Angew. Chem., Int. Ed. 2007, 46, 1018-1025.

14. Sumerlin, B. S.; Tsarevsky, N. V.; Louche, G.; Lee, R. Y.; Matyjaszewski, K. Macromolecules 2005, 38, 7540-7545.

15. Matyjaszewski, K.; Xia, J. Chem. Rev. 2001, 101, 2921-2990.

16. Wang, J.-S.; Matyjaszewski, K. J. Am. Chem. Soc. 1995, 117, 5614-5615.

17. Matyjaszewski, K.; Tsarevsky, N. V. Nat. Chem. 2009, 1, 276-288.

18. Lutz, J.-F.; Börner, H. G.; Weichenhan, K. Macromol. Rapid Commun. 2005, 26, 514-518. 